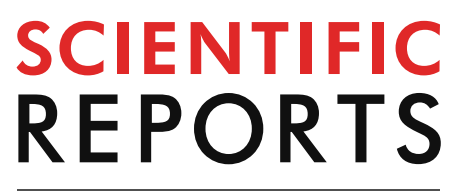

natureresearch

Check for updates

\title{
Age-specific modulation of intermuscular beta coherence during gait before and after experimentally induced fatigue
}

\author{
Paulo Cezar Rocha dos Santos ${ }^{1,2,5 凶}$, Claudine J. C. Lamoth ${ }^{1}$, Fabio Augusto Barbieri ${ }^{3}$, \\ Inge Zijdewind ${ }^{4}$, Lilian Teresa Bucken Gobbi ${ }^{2}$ \& Tibor Hortobágyi ${ }^{1}$
}

We examined the effects of age on intermuscular beta-band $(15-35 \mathrm{~Hz})$ coherence during treadmill walking before and after experimentally induced fatigue. Older $(n=12)$ and younger $(n=12)$ adults walked on a treadmill at $1.2 \mathrm{~m} / \mathrm{s}$ for $3 \mathrm{~min}$ before and after repetitive sit-to-stand, rSTS, to induce muscle fatigability. We measured stride outcomes and coherence from 100 steps in the dominant leg for the synergistic (biceps femoris (BF)-semitendinosus, rectus femoris (RF)-vastus lateralis (VL), gastrocnemius lateralis (GL)-Soleus (SL), tibialis anterior (TA)-peroneus longus (PL)) and for the antagonistic (RF-BF and TA-GL) muscle pairs at late swing and early stance. Older vs. younger adults had 43-62\% lower GL-SL, RF-VL coherence in swing and TA-PL and RF-VL coherence in stance. After rSTS, RF-BF coherence in late swing decreased by $~ 20 \%$ and TA-PL increased by $16 \%$ independent of age $(p=0.02$ ). Also, GL-SL coherence decreased by $\sim 23 \%$ and increased by $\sim 23 \%$ in younger and older, respectively. Age affects the oscillatory coupling between synergistic muscle pairs, delivered presumably via corticospinal tracts, during treadmill walking. Muscle fatigability elicits age-specific changes in the common fluctuations in muscle activity, which could be interpreted as a compensation for muscle fatigability to maintain gait performance.

Healthy aging modifies the integrated and coordinated neural control of gait ${ }^{1,2}$. The age-related modifications in neuromuscular control are evidenced, among others, by the $40-50 \%$ increases in rectus (RF)-biceps femoris (BF) coactivation at heel strike during gait ${ }^{3-5}$. These alterations in neuromuscular control during gait are usually associated with age-related reductions in mechanical output at the ankle and increases in mechanical work generation at the hip joint ${ }^{6}$. Age-specific decreases in inhibitory cortical control are accompanied by decreases in Ia afferent input ${ }^{7}$ that may lead to a depression of the inputs from muscle afferents compensated by increases in coactivation between agonist and antagonistic muscles during gait ${ }^{3,4}$. Such reductions in inhibitory cortical control impair cortico-muscular communication by reducing the strengths of inputs via the corticospinal tract during muscle contraction ${ }^{8}$, indirectly reflected by changes in muscular coherence during walking ${ }^{1,9,10}$.

Intermuscular coherence is a correlational measure signifying the strength in the frequency domain between two signals recorded from two muscles, suggesting the oscillatory synchronization of action potentials discharged by motor units ${ }^{1,11}$. This oscillatory synchronization is indirectly related to common presynaptic inputs to the motor neuron pool of two or more muscles ${ }^{12-14}$, as an attempt by the central nervous system (CNS) to reduce the

\footnotetext{
${ }^{1}$ Department of Human Movement Sciences, University Medical Center Groningen, University of Groningen, Groningen, The Netherlands. ${ }^{2}$ Posture and Gait Studies Laboratory (LEPLO), Institute of Biosciences, Graduate Program in Movement Sciences, São Paulo State University (UNESP), Rio Claro, Brazil. ${ }^{3}$ Department of Physical Education, Human Movement Research Laboratory (MOVI-LAB), Graduate Program in Movement Sciences, São Paulo State University (UNESP), Bauru, Brazil. " Department of Biomedical Sciences of Cells and Systems, University of Groningen, University Medical Center Groningen, Groningen, The Netherlands. ${ }^{5}$ Present address: Department of Human Movement Sciences, University of Groningen, University Medical Center Groningen, A. Deusinglaan 1, 9713 AV Groningen, The Netherlands. ${ }^{\bowtie}$ email: paulocezarr@hotmail.com
} 


\begin{tabular}{|l|l|l|r|}
\hline & Younger & Older & p value \\
\hline Groups' characteristics \\
\hline $\mathrm{n}$ (Male) & $12(7)$ & $12(7)$ & \\
\hline Age (range), y & $22(20-25)$ & $71(66-77)$ & $<0.01$ \\
\hline Height, cm & $177.45 \pm 2.65$ & $173.13 \pm 2.22$ & 0.17 \\
\hline Body mass, kg & $69.81 \pm 3.29$ & $73.92 \pm 2.93$ & 0.71 \\
\hline SPPB, scores & $12 \pm 0.00$ & $12 \pm 0.00$ & 1.00 \\
\hline MFI, scores & $38.18 \pm 2.71$ & $34.58 \pm 2.83$ & 0.52 \\
\hline STS & \multicolumn{5}{|l}{} \\
\hline Repetition & $583.3 \pm 50.12$ & $134.12 \pm 33.11$ & $<0.01$ \\
\hline Duration, min & $19.48 \pm 1.71$ & $4.47 \pm 1.15$ & \\
\hline
\end{tabular}

Table 1. Participants' characteristics and scores on questionnaires. Values are mean \pm SE. SPPB short physical performance battery, MFI multidimensional fatigue inventory, STS sit-to-stand.

dimensionality and complexity of control ${ }^{15}$. Data from neurological patients suggest that beta-band coherence in the range of 15-35 Hz emanates from cortical structures, including the sensorimotor cortex to control presynaptic inputs ${ }^{12,16}$. In older adults, there is diminished beta-band intramuscular coherence during swing phase, which may indicate a reduced common drive within the proximal and distal part of the tibialis anterior (TA) muscle $^{1}$, results consistent with data in hand muscles ${ }^{17}$. However, how healthy aging affects coherence among muscles crossing the ankle, knee and hip joints during such a functional task as gait has not yet been examined. Intermuscular coherence could provide information on age-related neuromuscular adaptation by inferring the oscillatory coupling of muscles spanning lower extremity joints.

Age-typical reductions in neuromuscular function are associated with an increase in effort to execute activities of daily living ${ }^{18}$. The high effort-demand can increase susceptibility to muscle fatigability during gait ${ }^{19}$. Muscle fatigability, i.e., a decline in the capacity to generate voluntary force, affects motor control ${ }^{20-23}$. A consequence of exercise-induced fatigability is a decrease in the dimensionality of the control ${ }^{24}$ by increasing common input to several muscles ${ }^{25-27}$, which would imply impaired motor coordination during complex tasks ${ }^{28}$. Indeed, exerciseinduced fatigability is likely to be related to changes in the excitability along the neural axis, which might increase the coupling of oscillatory neural inputs, reflecting in the common fluctuation in muscle activity across muscle pairs $^{25,26}$. Indeed, experimental data suggest $50-90 \%$ increase in beta-band cortico-muscular and muscularmuscular coherence during voluntary contraction ${ }^{28-30}$ that may evolve as a neural compensatory strategy for the fatigue-induced decline in performance in healthy younger adults $\mathrm{s}^{23,26,28,29,31}$. Because fatigue perturbs neuromuscular control, fatigability protocols have often been used to probe older adults' gait adaptability in response to this form of internal perturbations $\mathrm{s}^{32-36}$.

Repetitive sit-to-stand (rSTS) is a model to induce muscle fatigability in lower extremity muscles and probe gait adaptability ${ }^{37}$. Following bouts of rSTS, maximal voluntary force, the associated surface electromyographic (EMG) activity, and median frequency of muscles involved in gait can decrease up to $18 \%{ }^{35,38}$. However, previous studies found only weak evidence for age-related adaptations in stride metrics after fatigue ${ }^{35}$. The possibility exists that age-specific neural compensations to fatigue would minimize deterioration in stride metrics and preserve gait performance. In the present paper, we explore this hypothesis by further probing adaptive neural mechanisms through intermuscular coherence in the beta-band. Therefore, we determined the effects of age on beta-band coherence during treadmill walking before and after experimentally induced fatigability. Based on age-typical distal to proximal reductions in mechanical work generation during treadmill walking ${ }^{6}$, we hypothesized that older compared with young adults would exhibit lower coherences in ankle muscles and higher coherences in muscles involved in hip flexion and extension. Concerning rSTS effects, we expected to find that muscle fatigability would strengthen coherence more in healthy younger compared with older adults in synergistic and antagonistic muscles around the ankle and knee joints during gait.

\section{Results}

Participants characteristics and fatigability outcomes. Older and younger adults were not different in height, body mass, muscle mass, cognitive status, physical fitness, and trait level of perceived fatigability (Table 1).

Concerning muscle fatigability, older vs. younger performed $\sim 4.3 \times$ fewer STS repetitions $\left(\mathrm{T}_{22}: 7.40, \mathrm{p}<0.01\right)$ (Table 1), accompanied by an increase in RPE from $\sim 8$ to $\sim 18$ in the two groups $\left(\mathrm{F}_{1.22}: 346.24, \mathrm{p}<0.01, \eta_{p}^{2}: 0.94\right.$, $\mathrm{d}: 1.90)$ (Table 2). There were Age and Time main effects for MVIF $\left(\mathrm{F}_{1.22}: 10.38\right.$ and 32.07, $\mathrm{p}<0.01$ for all, $\eta_{p}^{2}: 0.32$ and 0.59 ) (Table 2). The Age main effect indicated that older compared to younger adults were weaker $(\sim 37 \%$ lower MVIF, d: 0.82). After rSTS, MVIF decreased in the two age groups by $16 \%$ (d: 0.37 ).

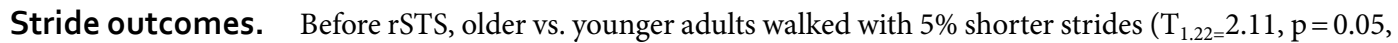
$\mathrm{d}: 0.86)$ and $5 \%$ higher cadence $\left(\mathrm{T}_{1.22=} 2.16, \mathrm{p}=0.04, \mathrm{~d}: 0.88\right)$ (Table 2$)$.

After rSTS, swing time decreased by $3 \%(\mathrm{p}<0.05, \mathrm{~d}: 0.40)$ but rSTS did not affect any other stride metrics. 


\begin{tabular}{|c|c|c|c|}
\hline & \multirow[b]{2}{*}{ Time } & Young adults & Older adults \\
\hline & & Mean \pm SE & Mean \pm SE \\
\hline \multicolumn{4}{|c|}{ Fatigability outcomes } \\
\hline \multirow{2}{*}{ MVIF, N } & Before rSTS & $676.25 \pm 244.62$ & $422.12 \pm 133.13^{\mathrm{a}}$ \\
\hline & After rSTS & $563.78 \pm 197.91^{\mathrm{b}}$ & $354.83 \pm 116.34^{\mathrm{a}, \mathrm{b}}$ \\
\hline \multirow{2}{*}{ Borg, score } & Before rSTS & $6.75 \pm 1.22$ & $8.42 \pm 2.43$ \\
\hline & After rSTS & $19.25 \pm 2.30^{\mathrm{b}}$ & $18 \pm 1.91^{\mathrm{b}}$ \\
\hline \multicolumn{4}{|l|}{ Stride outcomes } \\
\hline \multirow{2}{*}{ Stride length, $\mathrm{cm}$} & Before rSTS & $132.67 \pm 2.46$ & $123.97 \pm 2.23^{\mathrm{a}}$ \\
\hline & After rSTS & $130.25 \pm 2.17$ & $123.63 \pm 2.61$ \\
\hline \multirow{2}{*}{ Width, cm } & Before rSTS & $10.19 \pm 1.30$ & $10.3 \pm 0.89$ \\
\hline & After rSTS & $10.17 \pm 1.28$ & $10.21 \pm 0.76$ \\
\hline \multirow{2}{*}{ Swing time, $\mathrm{s}$} & Before rSTS & $0.39 \pm 0.01$ & $0.37 \pm 0.01$ \\
\hline & After rSTS & $0.38 \pm 0.01^{\mathrm{b}}$ & $0.37 \pm 0.01$ \\
\hline \multirow{2}{*}{ Stance time, $\mathrm{s}$} & Before rSTS & $0.72 \pm 0.04$ & $0.67 \pm 0.04$ \\
\hline & After rSTS & $0.71 \pm 0.04$ & $0.67 \pm 0.05$ \\
\hline \multirow{2}{*}{ Cadence, steps/min } & Before rSTS & $108 \pm 2.00$ & $114 \pm 2.00^{\mathrm{a}}$ \\
\hline & After rSTS & $109 \pm 2.00$ & $114 \pm 2.00$ \\
\hline
\end{tabular}

Table 2. Effects of age and rSTS on stride outcome. Values are mean \pm SE. ${ }^{a}$ Older $\neq$ Younger adults; ${ }^{\mathrm{b}}$ After $\neq$ Before rSTS.
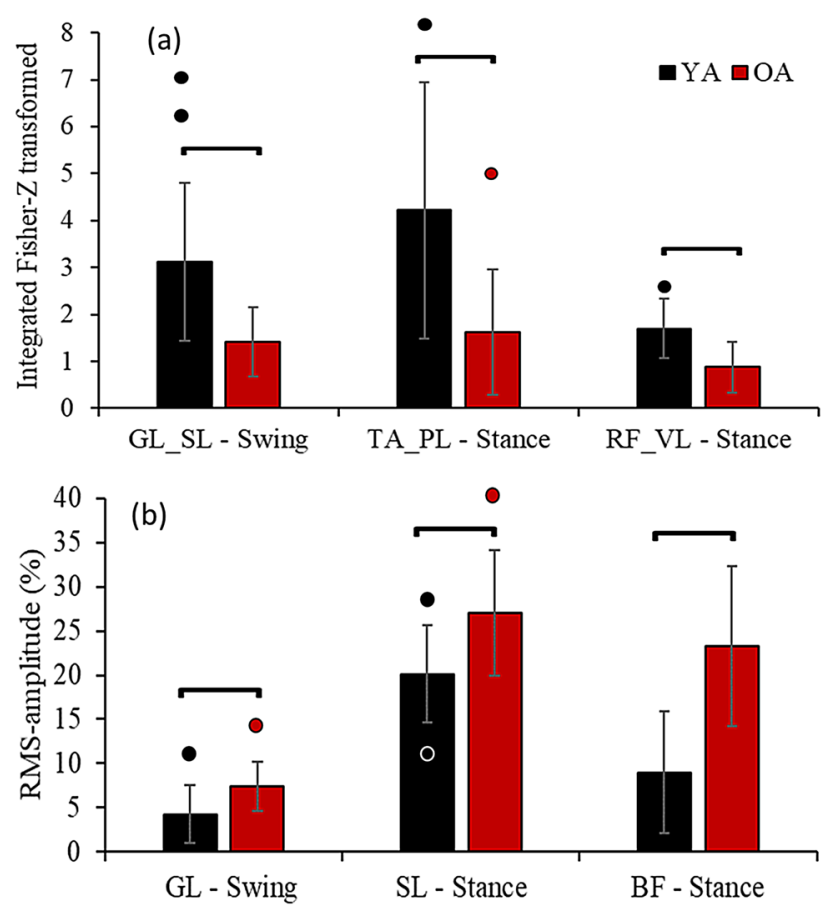

Figure 1. Mean \pm standard deviation for the significant outcomes (dots are outliers). (a) Intermuscular coherence during walking between gastrocnemius lateralis (GL)-Soleus (SL) in swing phase and between tibialis anterior (TA)-peroneus longus (PL) and rectus femoris (RF)-vastus lateralis in stance phase. (b) RMS-amplitude for GL in swing, and SL and biceps femoris (BF) both in stance. YA younger adults, OA older adults.

Coherence and EMG-amplitude. ${ }^{*}$ Supplementary Figures S1 and S2 detail the coherence data for all muscles and the two age groups before and after rSTS.

Before rSTS, older vs. younger adults had $\sim 55 \%$ lower GL-SL beta-band coherence $\left(\mathrm{t}_{22}: 3.59, \mathrm{p}<0.01, \mathrm{~d}: 1.32\right)$ in late swing and $62 \%$ and $48 \%$ lower TA-PL $\left(\mathrm{t}_{22}: 2.53, \mathrm{p}=0.02, \mathrm{~d}: 1.21\right)$ and RF-VL coherence $\left(\mathrm{t}_{22}: 3.42, \mathrm{p}<0.01\right.$, d: 1.41) in early stance (Fig. 1a). Concerning RMS-amplitude, older vs. younger adults had 75, 34 and $158 \%$ greater amplitude of GL in swing $\left(\mathrm{t}_{22}: 3.11, \mathrm{p}<0.01, \mathrm{~d}: 1.05\right)$, and SL $\left(\mathrm{t}_{22}: 2.65, \mathrm{p}<0.02, \mathrm{~d}: 1.09\right)$ and BF $\left(\mathrm{t}_{22}: 4.44\right.$, $\mathrm{p}<0.01$, d: 1.77) (Fig. 1b). 


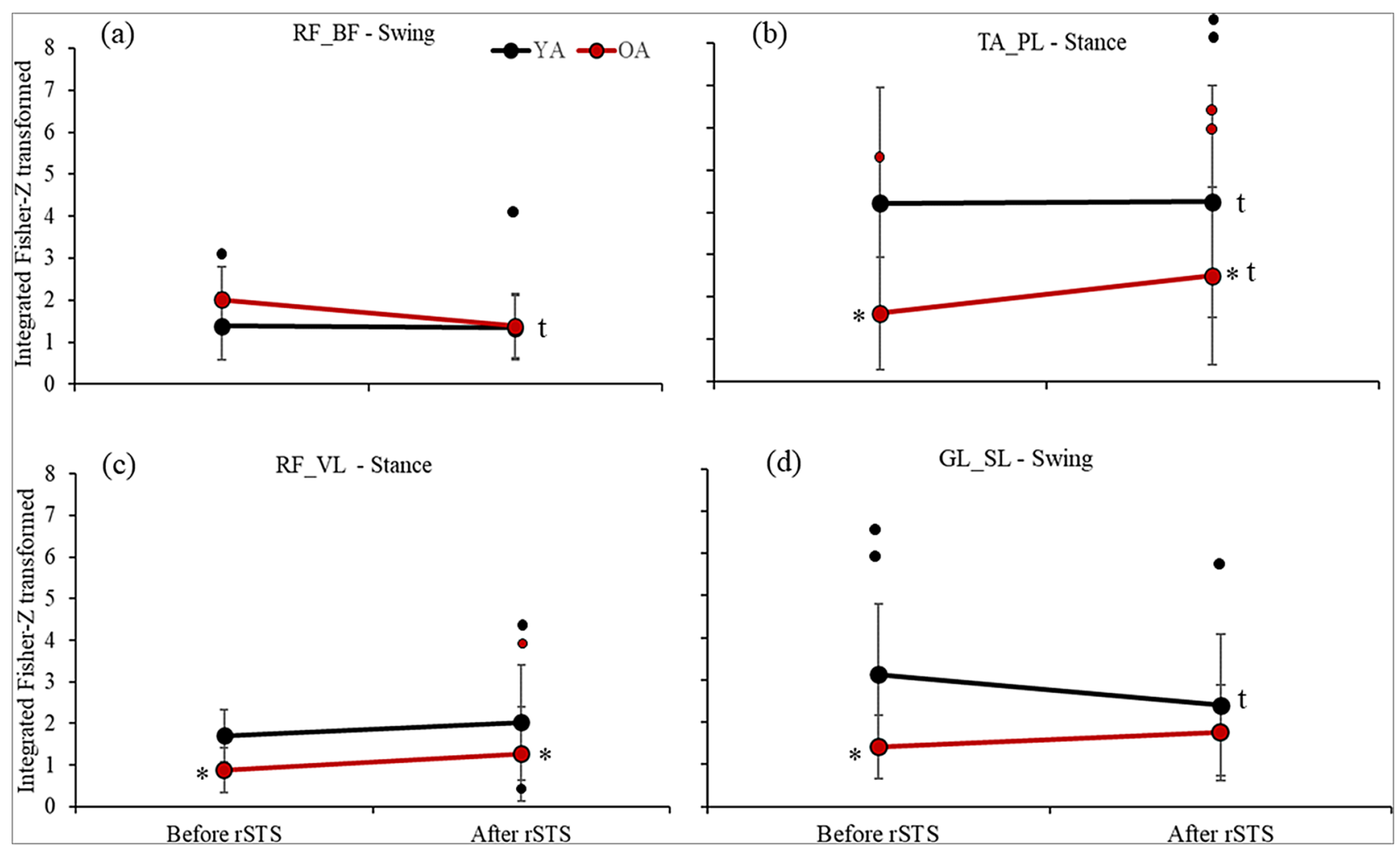

Figure 2. Mean \pm standard deviation (dots are outliers) for the significant outcomes. (a) Intermuscular coherence between RF-BF in swing, (b) TA-PL and (c) RF-VL in stance, and (d) GL-SL in swing phase during treadmill walking. ${ }^{\star}$ Age-differences [Older $(\mathrm{OA}) \neq$ Younger adults (YA)] indicated in post-hoc; time-differences $[$ After $\neq$ Before repetitive sit-to-stand (rSTS)] indicated by Post-hoc.

After rSTS, RF-BF coherence decreased $\left(\mathrm{F}_{1.22}: 6.68, \mathrm{p}=0.02, \eta_{p}^{2}: 0.23\right)$ in late swing and TA-PL coherence increased in early stance phases $\left(\mathrm{F}_{1.22}: 6.45, \mathrm{p}=0.02 ; \eta_{p}^{2}: 0.23\right)$. RF-BF coherence decreased by $20 \%$ in swing phase (d: 0.35 ) and TA-PL coherence increased by $16 \%$ in stance phase (d: 0.21 ) (Figs. 2a,b) in the two age groups combined (time main effect). After rSTS, older vs. younger had $\sim 43$ less RF-VL $\left(\mathrm{F}_{1.22}: 7.63, \mathrm{p}=0.01 ; \eta_{p}^{2}: 0.26 ; \mathrm{d}\right.$ : 0.86 ) (Fig. $2 \mathrm{c})$ and $44 \%$ less TA-PL coherences $\left(\mathrm{F}_{1.22}: 4.75, \mathrm{p}=0.04 ; \eta_{p}^{2}: 0.18 ; \mathrm{d}: 0.76\right)$ in stance phase.

There was a Group by Time effect for GL-SL coherence in swing phase $\left(\mathrm{F}_{1.22}: 5.03, \mathrm{p}=0.04 ; \eta_{p}^{2}: 0.19\right)$ so that older vs. younger adults had $55 \%$ lower coherence at the baseline $(\mathrm{p}<0.01 ; \mathrm{d}: 1.32)$, without group-difference after rSTS ( $p>0.05)$. This is because in younger adults, GL-SL coherence after rSTS decreased $(23 \%)(p=0.03$, d: 0.43 ), while older adults GL-SL coherence after rSTS increased (23\%, n.s.) (Fig. 2d).

For RMS-amplitude, ANOVA revealed time main effect indicating that both age groups combined decreased $(\sim 19$ and $17 \%)$ RMS of ST in both swing and stance phase $\left(\mathrm{F}_{1.22}: 5.87\right.$ and 6.89 , both $\mathrm{p}<0.03 ; \eta_{p}^{2}: 0.21$ and 0.24 ; $\mathrm{d}: 0.42$ and 0.26 , respectively) (Fig. $3 \mathrm{a}, \mathrm{b})$, and GL in stance phase $\left(\mathrm{F}_{1.22}: 4.49, \mathrm{p}<0.05 ; \eta_{p}^{2}: 0.17\right.$; d: 0.30) (Fig. 3c), and increased $(\sim 35 \%)$ the RMS of VL in stance phase $\left(\mathrm{F}_{1.22}: 8.22, \mathrm{p}<0.01 ; \eta_{p}^{2}: 0.27 ; \mathrm{d}: 0.73\right)$ (Fig. 3d).

Before rSTS, there were no correlations between beta-band coherence in GL-SL in late swing, TA-PL and RF-VL early stance with MVIF, stride length, and cadence, and between RMS-amplitude of GL with GL-SL both in late swing $(r=0.20$ to $0.38 ; \mathrm{p}>0.05)$ (Supplementary Figure S3). rSTS-induced decreases in GL-SL coherence during Swing phase correlated with decreases in swing time $(\mathrm{r}=0.59, \mathrm{p}<0.01)$ (Fig. 4). Absolute changes in variables did not correlate with absolute changes in coherence after $\mathrm{rSTS}(\mathrm{r}=0.02$ to $0.19, \mathrm{p}>0.05)$ (Supplementary Figure S4).

\section{Discussion}

We examined the effects of age on beta-band coherence during treadmill walking before and after experimentally induced muscle fatigability. The data confirmed the hypothesis of age-specific reductions in beta-band coherence between specific ankle muscles during gait. Against the hypothesis, we did not find the expected age-related increases in coherence among hip muscles. Also against the hypothesis, experimentally induced muscle fatigability did not strengthen the beta-band coherence across synergistic and antagonistic muscle pairs around the ankle and knee joints during gait in an age-specific manner. We, however, observed that older adults developed a compensatory neural strategy by increasing the TA-PL and GL-SL beta-band coherence in response to experimentally induced fatigability. Together, age affects the oscillatory coupling between synergistic muscle pairs, delivered presumably via corticospinal tracts, during treadmill walking. Muscle fatigability elicits age-specific changes in the common fluctuations in muscle activity, which could be interpreted as a compensation for muscle fatigability to maintain gait performance. 

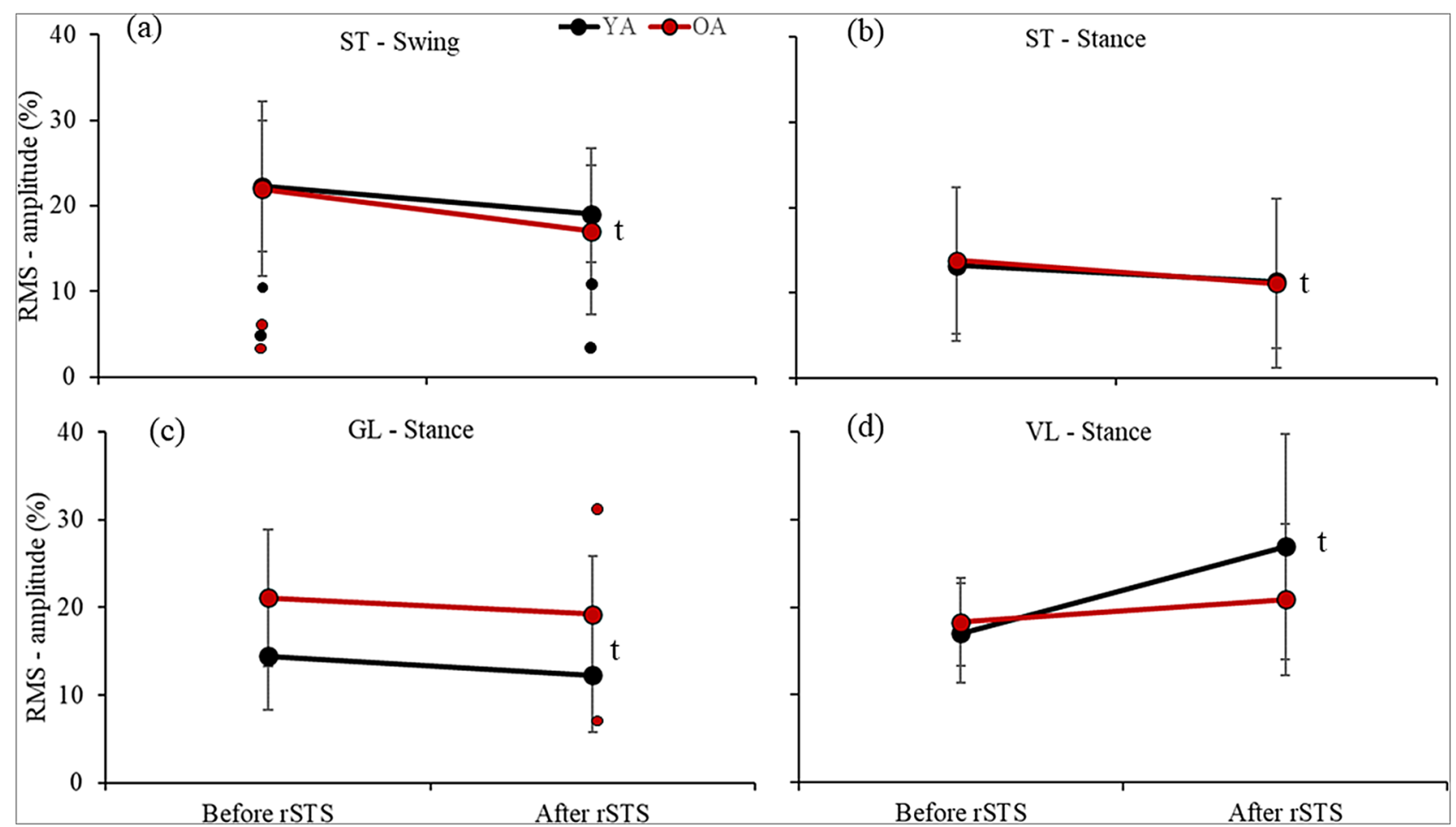

Figure 3. Mean \pm standard deviation (dots are outliers) for the significant RMS-amplitude normalized by maximum amplitude on stride cycle. (a) ST in swing, (b) ST in stance, (c) GL in stance, and (d) VL in stance.; time-differences [After $\neq$ Before repetitive sit-to-stand (rSTS)] indicated by Post-hoc.

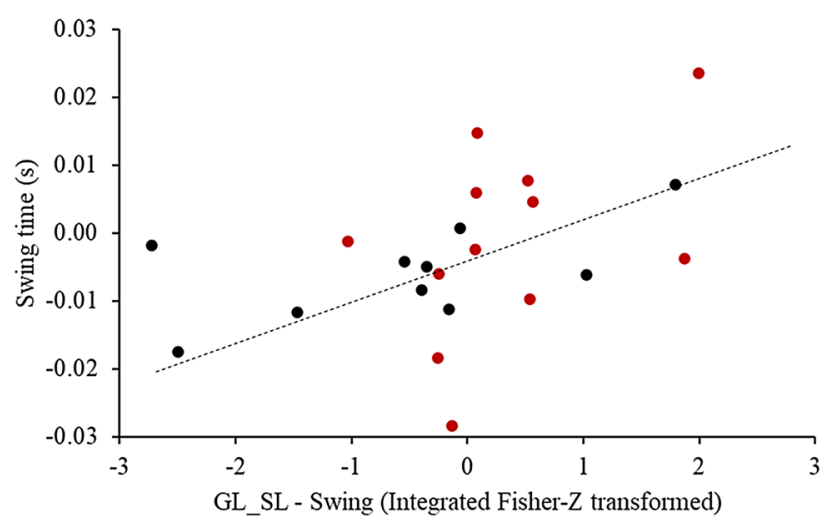

Figure 4. Significant correlations between absolute changes in GL-SL in swing phase and swing time due to muscle fatigability.

Concerning the age-effects on beta-band coherence during treadmill walking, we found that beta-band coherence was $44-62 \%$ lower in TA-PL, GL-SL, and RF-VL in older vs. younger adults during gait (Fig. 1). The ageeffects on intermuscular coherence agree with previous intramuscular coherence data indicating an age-typical reduction in coherence between proximal and distal $\mathrm{TA}^{1,9}$. However, our data extend those previous findings ${ }^{1,9}$ by showing age-related reductions in coherence between synergistic ankle and knee muscle pairs. The age-related reductions in coherence GL-SL, TA-PL, and RF-VL may be related to the age-typical decline/weakness of common presynaptic inputs to the motor neuron pool ${ }^{17}$ that potentially reflected in a reduced oscillatory behavior between muscles activated during walking ${ }^{1,10,39,40}$. Indeed, brain data suggest enhanced inhibitory cortical control in old age that is associated with reduced oscillatory activation of motoneurons ${ }^{41}$. The inhibitory control may reflect the number and strength of neural commands from the cortex to the muscles, as confirmed by the reduced cortico-muscular coherence ${ }^{1,39}$. Because it is widely believed that intermuscular beta-band coherence may arise from shared inputs via branched corticospinal pathways ${ }^{16,17,39,42}$, reduced beta-band coherence in older adults might be indicative of inefficiency in the CNS to reduce the dimensionality by sending common drives to muscles that exert similar functions ${ }^{17}$. The reductions in beta-band coherence, therefore, may reflect a decline in motor performance ${ }^{43}$. Indeed, the reduced beta-band coherence in the ankle and knee synergistic extensor muscles in 
older adults were accompanied by altered stride pattern, i.e., $\sim 10 \mathrm{~cm}$ shorter stride length and 6 steps $/ \mathrm{min}$ higher cadence (Table 2), although the correlation failed in showing any association.

Curiously, our results indicated age-differences only for synergistic (BF-VL, TA-PL, and GL-SL, Fig. 2) but not for antagonistic muscle pairs coherence (RF-BF and TA-PL). Such findings only in synergistic and not in antagonistic muscle pairs are reasonable ${ }^{44}$, considering synergistic/antagonistic muscle cooperation during the gait cycle. For example, the gastrocnemius and soleus act together to stabilize the ankle and to absorb the energy generated by the braking impulse at heel strike ${ }^{45}$. Reduced beta-band coherence in these muscles can imply in event-related desynchronization during gait, implying less muscle coordination to stabilize the ankle joint during the braking impulse ${ }^{46}$. The data also suggest a reduced oscillatory synchronization for TA and PL (62\%) during early stance phase (Fig. 2) and for RF and VL (48 and 43\%) in both late swing and early stance phases in older compared with younger adults (Figs. 1, 2b,c). These two muscle pairs work to decelerate the center of mass and stabilize the joints, keep the toe-clearance, and maintain the natural stance movement mechanics ${ }^{45}$. A lack of coherence for the antagonistic RF-BF and TA-GL muscle pairs might suggest that independent neural commands are being delivered to each muscle. This idea is further supported by previous data showing lower intermuscular coherence in antagonistic vs. synergistic muscle pairs ${ }^{44,47}$, and by studies that indicate that such antagonistic muscles activate in different moments of the gait cycle ${ }^{45}$. Although we observed age-related changes in beta-band coherence in synergistic muscles, there was an absence of accentuated antagonistic intermuscular beta-band coherence in healthy older adults, such as those observed in spinal cord injury population ${ }^{16}$. Possibly, it might be due to a relatively preserved pyramidal tract in healthy old vs. spinal cord injury population.

Our findings extend the current literature ${ }^{1,9}$ to infer an age-related decline in the oscillatory coupling between muscles spanning lower extremity joints (ankle, knee and hip). The age-related decrease in common neural input occurred in synergistic lower extremity muscles. Curiously, such decreases seem not to be related to the age-typical reductions in mechanical output at the ankle and the increases in mechanical work generation at the hip joint ${ }^{6}$. Reduced RF-VL, TA-PL, and GL-SL beta-band coherence in older vs. younger adults may be related to general age-related reductions in the common neural drive via corticospinal tracks to synergistic muscle pairs during treadmill walking. Such age-specific reductions in beta-band coherence between synergistic muscle pairs before rSTS were further modulated in order to compensate for the muscle fatigability in older adults, thus keeping the stride metrics of gait performance unchanged (Table 2).

With regards to the fatigability effects on beta-band coherence, we expected that muscle fatigability would strengthen coherence around the ankle and knee joints during gait more so in healthy younger than in older adults. We, however, observed an age-specific modulation intermuscular coherence in ankle muscle pairs after muscle fatigability. Such a modulation suggests that older adults developed a compensatory strategy in response to the decline in motor performance induced by rSTS to maintain neural control of lower extremity muscles during gait, resulting in unchanged stride metrics of gait performance.

Muscle fatigability tends to increase intermuscular beta-band coherence ${ }^{25,26,28}$. This increase may be interpreted as a compensatory mechanism of CNS to increase the strength of the common neural drive to muscles to maintain a target force or performance ${ }^{26,28}$. Therefore, our result of subtle increases in TA-PL beta-band coherence in stance phase is in line with the expected changes in coherence after rSTS. In contrast and unexpectedly, both age groups revealed a slight decrease in RF-BF intermuscular coherence in the stance phase. Additionally, the particular age adaptation in synergistic GL-SL intermuscular coherence in stance phase was also unforeseen.

Perhaps, the most intriguing result was the age-specific adaptation in intermuscular coherence in ankle muscle pairs after muscle fatigability. While there was $23 \%$ decrease in GL-SL common drive in younger adults, older adults showed $23 \%$ increase in this coherence after muscle fatigability (Fig. $2 \mathrm{~d}$ ). In addition, the main effect indicated a $16 \%$ increase in TA-PL coherence in the two age groups after rSTS, driven by the 55 vs. $1 \%$ increase in older and younger adults (Fig. 2b). Thus, there was a trend for age by time interaction in this important outcome $\left(p=0.075 ; \eta_{p}^{2}: 0.14\right)$. One possible interpretation for age-differences in these intermuscular coherences is compensation for the decline in motor performance induced by rSTS. Age-specific increase in these coherences may reflect higher susceptibility to fatigability ${ }^{19}$ and/or an age-related increase in demand of walking after muscle fatigability $^{5}$. Indeed, while both age groups showed similar decreases in MVIF, older compared with younger adults performed remarkably fewer STS repetitions (Table 2).

Maximal voluntary contraction-induced fatiguability was associated with physiological tremor, which was interpreted as an increase in afferent feedback from muscle spindles to the motor units ${ }^{30}$. The increase in feedback presumably strengthens the correlated inputs to motor units as fatigability progresses ${ }^{30}$. Our results partially support this finding because while we observed increases in beta-band coherence, the increase in alpha-band coherence was minimal as were the correlations between these two coherences in older adults (data not shown). Concerning the higher walking demand, previous data showed that the metabolic cost of gait was $20 \%$ higher, which was related to $67 \%$ higher agonist muscle activation and $153 \%$ greater antagonist coactivation during walking ${ }^{5}$. Together, the higher muscle fatigability effects and higher walking demand in older compared with younger adults seem to have elicited compensatory neural adaptations to muscle fatigability, making it possible for older adults to continue to walk.

The small decreases in intermuscular coherence between antagonist muscle pairs (RF-BF) were somewhat unexpected. Normally such coherences tend to increase rather than decrease after muscle fatigability. The increases are attributed to enhanced cortical excitability to compensate for muscle fatigability ${ }^{25,31}$, normally associated with higher coactivation. Although high cortical excitability due to muscle fatigability is a physiological explanation for both increases in muscle amplitude and coherence ${ }^{25,31}$, we recognize that increases in intermuscular coherence do not necessarily mean an increase in coactivation. In addition, both increased coactivation and antagonistic intermuscular coherence could impair muscle coordination and motor performance $e^{4,26}$.

The changes in beta-band coherence after experimentally induced fatigability were not accompanied by any meaningful changes in stride outcomes during treadmill walking (Table 2). Our results indicating only small 
changes in swing time $(0.01 \mathrm{~s})$ for young adults reiterate the minimal effects of age and fatigability on strides outcomes observed previously ${ }^{35}$. Possibly, those changes in beta-band coherence may represent a compensatory neural strategy in response to experimentally induced fatigability to maintain the strides metrics unchanged.

As limitations, albeit intermuscular coherence during gait has recently received attention, the interpretation of such data is not straightforward. It is because relating individual variation in intermuscular coherence to variation in gait behavior due to age or perturbations, as done here, has been moderately successful at best ${ }^{1,9}$. In addition, although our findings revealed large ANOVA effects sizes $\left(\eta_{p}^{2}\right)$ and significant effects in selected coherence outcomes, the statistical powers were lower (between 60 and 79\%) than the ideal levels of power (80\%) for such outcomes (details in Supplementary analysis), suggesting that the study might have been underpowered ${ }^{48}$. Furthermore, the influence of EMG rectification on coherence has been discussed ${ }^{46,49,50}$. We opted to use EMG rectification procedure because it amplifies temporal information about motor unit action potentials during submaximal tasks, as gait ${ }^{46,49-51}$, and helps with the interpretation of the results with the literature $\mathrm{e}^{1,10,39,42,46,47,52}$. The Fourier transformation (a standard protocol used in coherence analyses ${ }^{1,10,42,46}$ ) is valid for stationary signals, a condition unlikely to be met in gait. To address this limitation, we analyzed coherence in short time windows during swing and stance phases of gait. Similarity of temporal features of gait before and after fatigability in younger and older adults further decreased the likelihood of non-stationarity in the signal (Table 2). In addition, we recognize the limitation of coherence analyses because the cortical or spinal origin of coherence is not unambiguous and could be affected by amplitude cancellation and cross-talk ${ }^{53}$. However, we have made every effort to minimize such effects by computing RMS-amplitude and verifying the absence of correlations between absolute values and changes between RMS-amplitudes and coherence for the significant outcomes. While treadmill vs. overground walking has the advantage of examining gait under a standardized condition, treadmill walking requires reactive rather than feed-forward adjustments of walking. Treadmill makes walking unvarying ${ }^{54,55}$ and, compared with overground walking, it reduces muscle activation ${ }^{56}$ and coherence ${ }^{46}$. Therefore, the age and fatigability effects on intermuscular coherence might be more overt during overground walking.

The current study contributes to the understanding of how health aging brings about adaptations muscle activation during gait and how such adaptations might turn into compensations to help maintain gait performance on the treadmill. In conclusion, age affects the oscillatory coupling between synergistic muscle pairs, delivered presumably via corticospinal tracts, during treadmill walking. Muscle fatigability elicits age-specific changes in the common fluctuations in muscle activity, which could be interpreted as a compensation for muscle fatigability to maintain gait performance.

\section{Methods}

Participants. Healthy younger $(n=12$, age range $20-25$ years, $5 F)$ and older $(n=12$, age range $66-77$ years, $5 F$ ) participants completed the study. Inclusion criteria were: age $<25$ or $>65$ years and either gender. Exclusion criteria were: lower limb musculoskeletal injury or surgery that could affect walking ability; inability to walk unassisted on a treadmill; self-reported pain in the lower extremities, and neurological or cardiac diseases. The procedures of this study were performed in accordance with the Declaration of Helsinki ${ }^{57}$ and were approved by the Ethical Committee of the Department of Human Movement Sciences, University Medical Center Groningen (\#ECB2017.06.12_1). All participants signed the informed consent document before testing.

Procedures. The cognitive status, physical fitness, and trait level of perceived fatigability were assessed by the Mini-Mental State Examination ${ }^{58}$, Short Physical Performance Battery ${ }^{59}$, and Multidimensional Fatigue Inventory ${ }^{60}$, respectively. Participants then walked on a treadmill for $3 \mathrm{~min}$, performed a knee extension to measure the maximal voluntary isometric force (MVIF), executed the rSTS to induce muscle performance fatigability, performed an MVIF, and walked on the treadmill (Fig. 5), described in detail previously ${ }^{35}$.

Data acquisition. Participants, wearing a harness, walked on a treadmill instrumented with two force plates (Motek, Amsterdam, NL) for 3 min before and after rSTS. They were asked not to hold onto the handrails and to look at a target (letter X) displayed on the wall in front of them $5 \mathrm{~m}$ away. We assessed gait in an instrumented treadmills to be able to record consecutive steady-state stride cycles, exactly controlling walking speed ${ }^{61}$. Walking speed was fixed at $1.2 \mathrm{~m} / \mathrm{s}$. A fixed walking speed was chosen to avoid a potential effect of speed on intermuscular coherence, and $1.2 \mathrm{~m} / \mathrm{s}$ was selected to be similar to the comfortable speed for both young and older groups ${ }^{62,63}$. We collected 3-D ground reaction forces and moments of force under each leg at $1 \mathrm{kHz}$ using a D-Flow acquisition software (Motek, Amsterdam, NL, USA).

To record EMG activity during walking, 8 wireless sensors (dimensions: $37 \times 26 \times 15 \mathrm{~mm}$, electrode material: silver; Trigno Wireless System, Delsys, Natick, MA, USA) were placed unilaterally on the following muscles: soleus (SL), gastrocnemius lateralis (GL), TA, peroneus longus (PL), vastus lateralis (VL), rectus femoris (RF), biceps femoris (BF), and semitendinosus (ST) according to Surface Electromyography for the Non-Invasive Assessment of Muscles (SENIAM) conventions ${ }^{64}$. EMG signals were sampled at $2 \mathrm{kHz}$. The areas where the sensors were placed, body hair was removed, and the skin was cleaned with alcohol. Treadmill and EMG data acquisition were electronically synchronized with a custom-built timer and event generator.

MVIF was assessed on a custom-built dynamometer. Participants, seated in a chair with the knee and hip in $90^{\circ}$ of flexion (measured by a Goniometer), had the non-dominant lower leg strapped to the chairs' lever arm ( $\sim 10 \mathrm{~cm}$ up to lateral malleolus). We instructed participants to contract the quadriceps as rapidly and forcefully as possible and to maintain force generation for $5 \mathrm{~s}$. MVIF was determined as the peak of force.

To induce muscle fatigability, participants performed the rSTS at $0.5 \mathrm{~Hz}$ in a standard dimension chair $(0.43 \times 0.41 \times 0.42 \mathrm{~m})$. The protocol was stopped either when participants were unable to continue or after $30 \mathrm{~min}$. 
Treadmill Walking

(a)

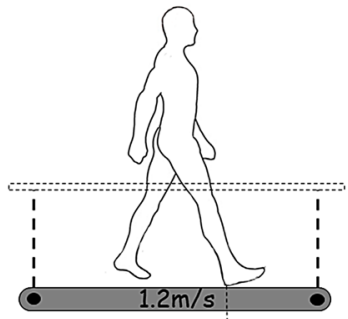
Heel Strike $(H S)$
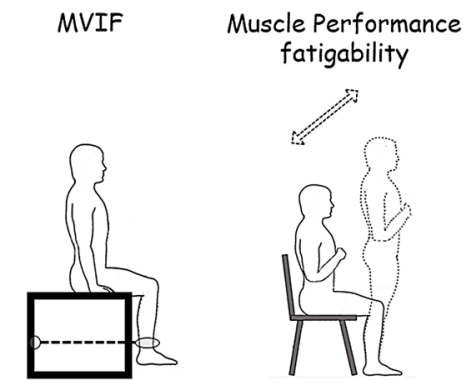
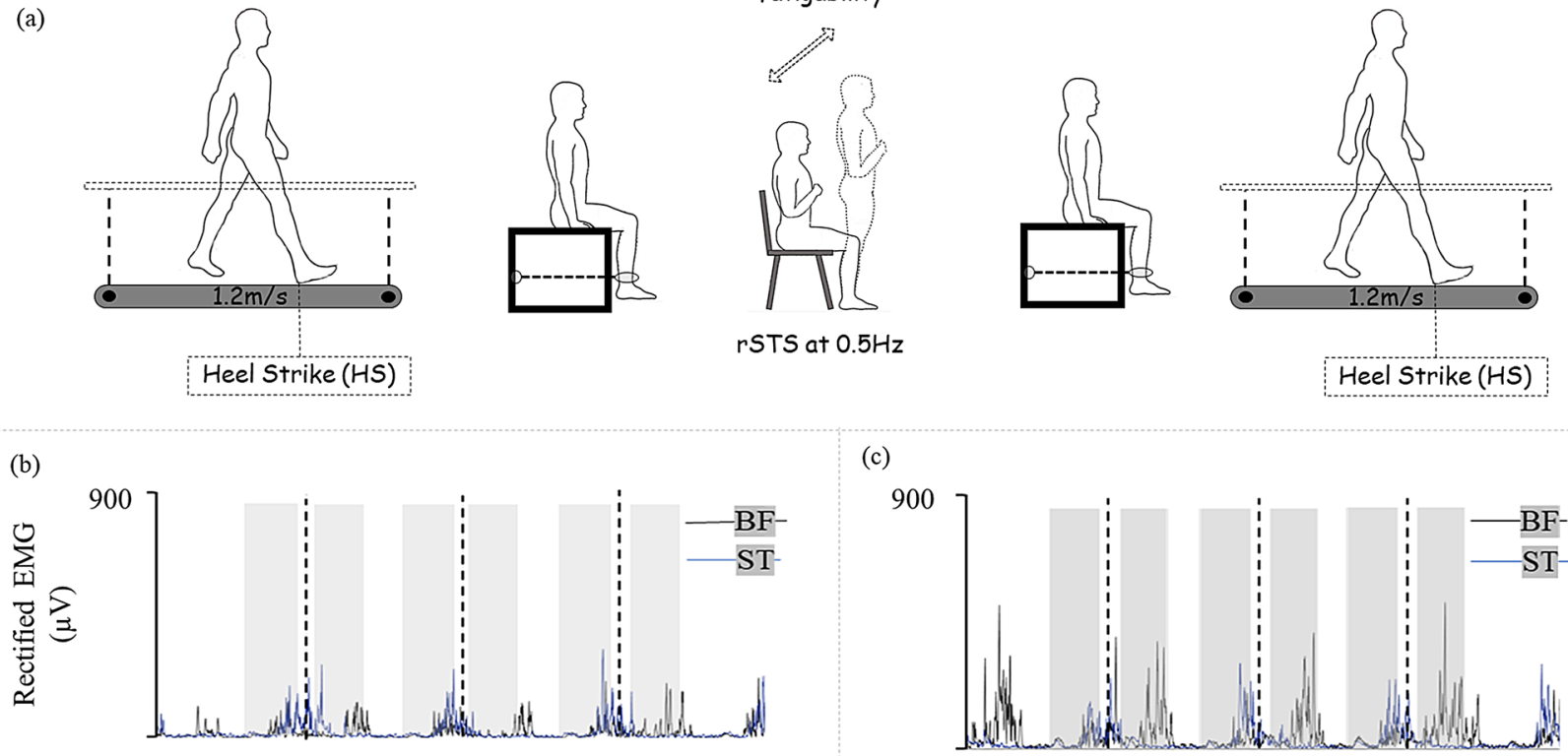

(c)
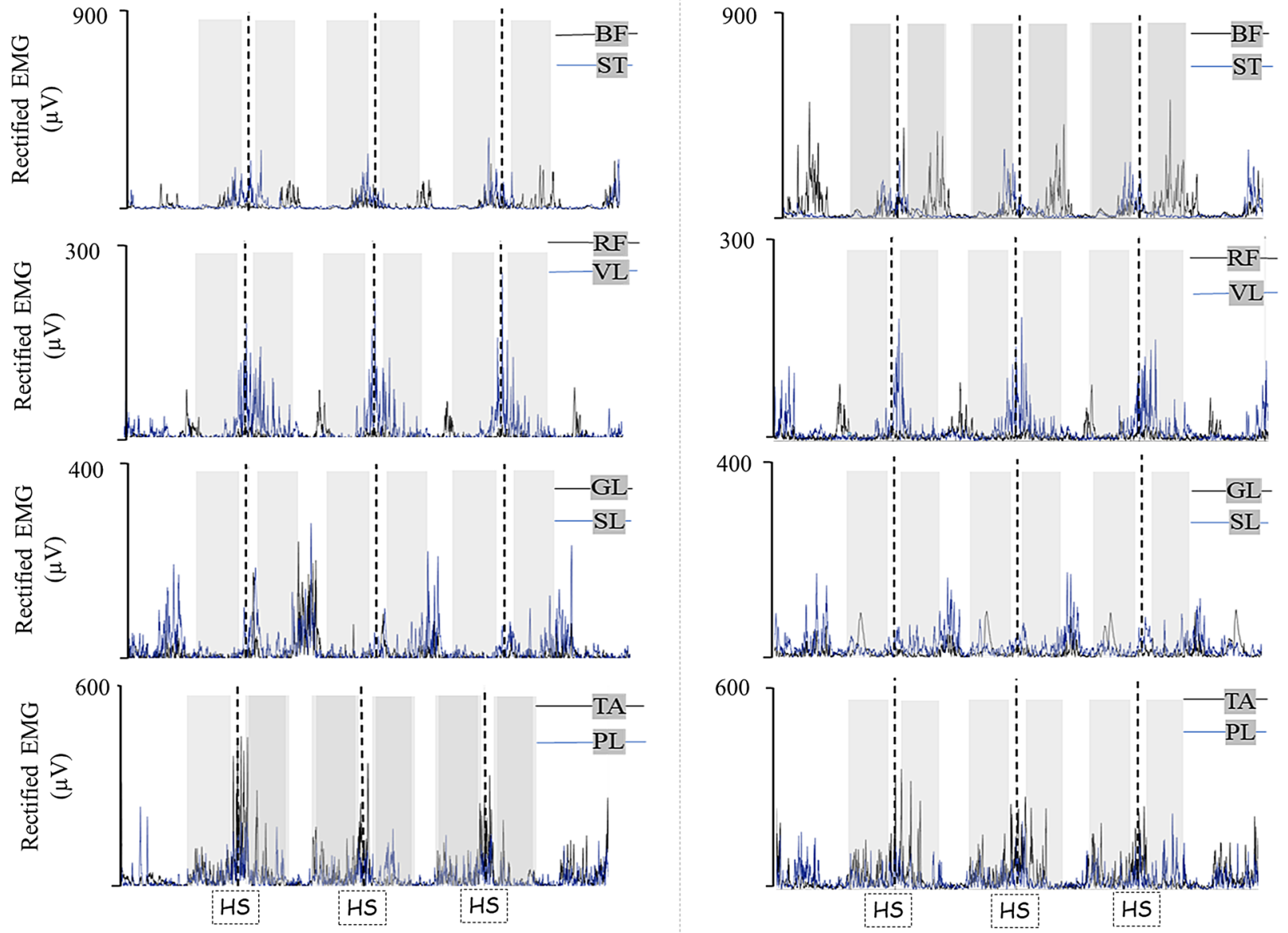

Figure 5. (a) Experimental setup and EMG data (data high-pass filtered $5 \mathrm{~Hz}$ using a second-order Butterworth filter, full-wave rectified using Hilbert transform data) of one younger subject before (b) and after (c) rSTS, considering 3 out of 100 heel strike (HS) events (dashed lines). Each plot on panel (b,c) consists of two muscles (represented in black and blue lines). Shaded areas illustrate the $350 \mathrm{~ms}$ of analyses for late swing $(-400$ to $-50 \mathrm{~ms}$ before heel strikes) and early stance (50-400 ms after heel strikes) phases. MVIF maximum voluntary isometric force, $r S T S$ repetitive sit-to-stand task, $H S$ heel strike, $B F$ biceps femoris, $S T$ semitendinosus, $R F$ rectus femoris (RF), $V L$ vastus lateralis, $G L$ gastrocnemius lateralis, $S L$ soleus, $T A$ tibialis anterior, $P L$ peroneus longus (PL).

Task duration and the number of repetitions were recorded. Before and after rSTS, we asked participants to rate their perceived exertion (RPE) on a 6-20 Borg Scale ${ }^{65}$.

Data analysis. Data were analyzed using custom MATLAB routines (version r2018; MathWorks, Natick, MA, USA). Ground reaction forces and moments of force were filtered with a $15-\mathrm{Hz}$ low-pass second-order zero-phase Butterworth filter. Heel contact and toe-off were determined using a threshold of $50 \mathrm{~N}$ vertical ground reaction force. Using heel strike and toe-off, we calculated stride length, step width, stance time, swing 
time, and cadence ${ }^{66}$ using the average of the middle 100 strides performed with the dominant leg during 3 min of treadmill walking before and after rSTS.

EMG data were visually inspected for minimizing noise and artifacts. Then, the data were high-pass filtered at $5 \mathrm{~Hz}$ using a second-order Butterworth filter, full-wave rectified using Hilbert transform. Then, EMG data were downsampled to $1 \mathrm{kHz}$ to obtain the same frequency of the force data plate.

Coherence calculation. We calculated the frequency-domain coupling between two EMG signals acquired during the treadmill walking before and after rSTS. For EMG coherence, we set two windows of $350 \mathrm{~ms}$ for analysis ${ }^{1}$ : late swing phase (from -400 to $-50 \mathrm{~ms}$ before the heel strike) and early stance phase (from 50 to $350 \mathrm{~ms}$ after the heel strike) (Fig. 5b,c). In both phases, we calculated coherence considering 4 synergistic muscle pairs: BF-ST, RF-VL, GL-SL, TA-PL, and 2 antagonistic muscle pairs: RF-BF; TA-GL.

To calculate intermuscular coherence, we first determine the auto-spectra of each muscle $\left(f_{x x}\right.$ and $\left.f_{y y}\right)$ and cross-spectrum $\left(f_{x y}\right)$ of the muscle pairs (TA-PL, GL-SL, RF-VL, BF-ST, TA-GL, and RF-BF) using Welch's periodogram method ${ }^{25,67}$. For each of the 100 strides selected, estimates were obtained using $350 \mathrm{~ms}$ window (separately for late swing and early stance phases), nonoverlapping data segments, resulting in a frequency of $2.86 \mathrm{~Hz}$ of resolution. Spectral estimates of individual strides were then averaged across the 100 strides (same used to calculate the stride outcomes) and used to calculate coherence. Intermuscular coherence was calculated by the squared modulus of cross-spectrum divided by the product of the two auto-spectrum for each frequency $(\lambda)^{11}$ :

$$
c(\lambda)=\frac{\left|f_{x y}(\lambda)\right|^{2}}{f_{x x}(\lambda) \cdot f_{y y}(\lambda)}
$$

where $f_{x x}$ and $f_{y y}$ are the auto-spectra of each muscle, and $f_{x y}$ is the cross-spectrum of the muscle pairs (TA-PL, GL-SL, RF-VL, BF-ST, TA-GL, and RF-BF). Values of coherence range from 0 (absent) to 1 (completely correlated $)^{11}$ and in a frequency range of $0-55 \mathrm{~Hz}$. For each subject, intermuscular coherence was significant when it exceeded the confidence limit for the number of segments $(\mathrm{L})$ used to estimate the spectrum ${ }^{11,68}$, as given:

$$
1-(\alpha)^{\frac{1}{L-1}}
$$

where $\alpha=0.05$ and $\mathrm{L}$ is the number of strides $(\mathrm{N}=100)$ used in the analysis.

For each subject and muscle pair, we ascertained from the cumulant density plots that high coherences were accompanied by near zero-lag synchronization suggesting that cross-talk did not affect coherences ${ }^{16,69}$. To compare coherence between age groups and time (before and after rSTS), intermuscular coherence of each subject was combined into pooled estimates for age groups for each walking phase before and after rSTS. Coherence estimates were Fisher transformed before pooling to stabilize variance ${ }^{68,69}$. The amount of coherence was calculated by the cumulative sum (area) on the range of the beta-band frequency domain $(15-35 \mathrm{~Hz})$ for each age group, phase, and before and after rSTS.

RMS-amplitude. For EMG amplitude, the raw EMG data were band-pass filtered $(20-450 \mathrm{~Hz})$ using a fourth-order Butterworth filter, full-wave rectified using Hilbert transform, and downsampled to $1 \mathrm{kHz}$. For each stride, the RMS-amplitude for each muscle was calculated considering the $350 \mathrm{~ms}$ windows at late swing and early stance phase, and the values were expressed as a percentage of the peak amplitude within the stride cycle considering the walking condition before rSTS. RMS-amplitudes were averaged across the 100 strides.

Statistical analysis. Statistical analyses were performed in SPSS for Windows (Version 25, IBM, Armonk, NY, USA). When Shapiro-Wilk tests revealed non-normal distribution, data were log-transformed for further comparisons using t-test or ANOVA. T-tests were used to compare the effects of age on Groups' characteristics (age, height, body mass, SPPB, MFI), rSTS duration, and the number of STS repetitions, stride outcomes, RMSamplitude, and beta-band coherence before rSTS. To compare the effects of experimentally induced fatigability, we conducted a repeated-measures ANOVA with as between factor Age (younger vs. older adults) and within factor Time (time 1: before rSTS vs. time 2: after rSTS) for MVIF, strides outcomes, RMS-amplitude, and betaband coherence. ANOVA effect size was estimated using partial eta squared $\left(\eta_{p}^{2}\right)$ with $\eta_{p}^{2}<0.01,0.06$ and $>0.13$ (that reflect in $f=0.1,0.25$ and $>0.40$ ) as small, medium and large effects size ${ }^{70}$. If interactions were significant, post-hoc comparisons for each factor were made, and the level of significance was adjusted for multiple comparisons by using Bonferroni correction. For post-hoc and t test comparisons, Cohen's d was calculated, and we interpreted $0.21-0.50,0.51$ to 0.79 and $>0.79$ as small, medium and large effect sizes (d), respectively ${ }^{70}$. In G-power ${ }^{71}$, post-hoc power analyses were calculated, with an assumed Type I error of 0.05 , considering the current sample size and the $\eta_{p}^{2}$ for each outcome. The power calculation resulted in a range of Type II error rate of $0.21-0.45$ (55-79\% statistical power).

As additional analyses, we computed correlations between the outcomes (beta-band coherence, MVIF, stride metrics). Before rSTS, a Spearman's correlation was computed between absolute values of the outcomes that indicated significant age differences $(\mathrm{p}<0.05)$. We also computed Spearman's correlations between absolute changes (delta, after-before rSTS) in the outcomes that revealed a significant Time effect. Additionally, if a $t$ test or ANOVA revealed significant differences in RMS-amplitude between two muscles included in coherence analyses, we computed Spearman's correlations between absolute values (before rSTS) and changes (delta) RMSamplitude and coherence. Such correlations would tell us if RMS-amplitude was driving coherence. 


\section{Data availability}

All data and additional figures generated in this study are included as Supplementary Information files.

Received: 14 May 2020; Accepted: 7 September 2020

Published online: 28 September 2020

\section{References}

1. Spedden, M. E., Choi, J. T., Nielsen, J. B. \& Geertsen, S. S. Corticospinal control of normal and visually guided gait in healthy older and younger adults. Neurobiol. Aging 78, 29-41 (2019).

2. Stuart, S., Alcock, L., Rochester, L., Vitorio, R. \& Pantall, A. Monitoring multiple cortical regions during walking in young and older adults: Dual-task response and comparison challenges. Int. J. Psychophysiol. 135, 63-72 (2019).

3. Lee, H.-J., Chang, W. H., Choi, B.-O., Ryu, G.-H. \& Kim, Y.-H. Age-related differences in muscle co-activation during locomotion and their relationship with gait speed: A pilot study. BMC Geriatr. 17, 44 (2017).

4. Hortobágyi, T. \& Devita, P. Mechanisms responsible for the age-associated increase in coactivation of antagonist muscles. Exerc. Sport Sci. Rev. 34, 29-35 (2006).

5. Hortobagyi, T., Finch, A., Solnik, S., Rider, P. \& De Vita, P. Association between muscle activation and metabolic cost of walking in young and old adults. J. Gerontol. Ser. A Biol. Sci. Med. Sci. 66, 541-547 (2011).

6. Waanders, J. B., Hortobágyi, T., Murgia, A., DeVita, P. \& Franz, J. R. Advanced age redistributes positive but not negative leg joint work during walking. Med. Sci. Sport. Exerc. 51, 615-623 (2019).

7. Papegaaij, S., Taube, W., Baudry, S., Otten, E. \& Hortobágyi, T. Aging causes a reorganization of cortical and spinal control of posture. Front. Aging Neurosci. 6, 28 (2014).

8. Matsuya, R., Ushiyama, J. \& Ushiba, J. Inhibitory interneuron circuits at cortical and spinal levels are associated with individual differences in corticomuscular coherence during isometric voluntary contraction. Sci. Rep. 7, 1-11 (2017).

9. Gennaro, F. \& Bruin, E. D. A pilot study assessing reliability and age-related differences in corticomuscular and intramuscular coherence in ankle dorsiflexors during walking. Physiol. Rep. 8, 20 (2020).

10. Roeder, L., Boonstra, T. W. \& Kerr, G. K. Corticomuscular control of walking in older people and people with Parkinson's disease. Sci. Rep. 10, 2980 (2020).

11. Halliday, D. M. et al. A framework for the analysis of mixed time series/point process data-Theory and application to the study of physiological tremor, single motor unit discharges and electromyograms. Prog. Biophys. Mol. Biol. https://doi.org/10.1016/S0079 -6107(96)00009-0 (1995).

12. Grosse, P., Cassidy, M. J. \& Brown, P. EEG-EMG, MEG-EMG and EMG-EMG frequency analysis: Physiological principles and clinical applications. Clin. Neurophysiol. 113, 1523-1531 (2002).

13. De Luca, C. J. \& Erim, Z. Common drive in motor units of a synergistic muscle pair. J. Neurophysiol. 87, 2200-2204 (2002).

14. Semmler, J. G., Kornatz, K. W. \& Enoka, R. M. Motor-unit coherence during isometric contractions is greater in a hand muscle of older adults. J. Neurophysiol. 90, 1346-1349 (2003).

15. Del Vecchio, A. et al. The human central nervous system transmits common synaptic inputs to distinct motor neuron pools during non-synergistic digit actions. J. Physiol. 597, 5935-5948 (2019).

16. Norton, J. A. \& Gorassini, M. A. Changes in cortically related intermuscular coherence accompanying improvements in locomotor skills in incomplete spinal cord injury. J. Neurophysiol. 95, 2580-2589 (2006).

17. Semmler, J. G., Kornatz, K. W., Meyer, F. G. \& Enoka, R. M. Diminished task-related adjustments of common inputs to hand muscle motor neurons in older adults. Exp. Brain Res. 172, 507-518 (2006).

18. Hortobágyi, T., Mizelle, C., Beam, S. \& DeVita, P. Old adults perform activities of daily living near their maximal capabilities. J. Gerontol. A. Biol. Sci. Med. Sci. 58, M453-M460 (2003).

19. Petrella, J. K., Kim, J., Tuggle, S. C., Hall, S. R. \& Bamman, M. M. Age differences in knee extension power, contractile velocity, and fatigability. J. Appl. Physiol. 98, 211-220 (2005).

20. Enoka, R. M. \& Duchateau, J. Translating fatigue to human performance. Med. Sci. Sport. Exerc. 48, 2228-2238 (2016),

21. Gandevia, S. C. Spinal and supraspinal factors in human muscle fatigue. Physiol. Rev. 81, 1725-1789 (2001).

22. Taylor, J. L., Butler, J. E. \& Gandevia, S. C. Changes in muscle afferents, motoneurons and motor drive during muscle fatigue. Eur. J. Appl. Physiol. 83, 106-115 (2000).

23. Fry, A., Mullinger, K. J., O’Neill, G. C., Brookes, M. J. \& Folland, J. P. The effect of physical fatigue on oscillatory dynamics of the sensorimotor cortex. Acta Physiol. (Oxf) 220, 370-381 (2017).

24. Monjo, F., Terrier, R. \& Forestier, N. Muscle fatigue as an investigative tool in motor control: A review with new insights on internal models and posture-movement coordination. Hum. Mov. Sci. 44, 225-233 (2015).

25. Boonstra, T. W. et al. Fatigue-related changes in motor-unit synchronization of quadriceps muscles within and across legs. J. Electromyogr. Kinesiol. 18, 717-731 (2008).

26. Semmler, J. G., Ebert, S. A. \& Amarasena, J. Eccentric muscle damage increases intermuscular coherence during a fatiguing isometric contraction. Acta Physiol. 208, 362-375 (2013).

27. Wang, L. et al. Fatigue-related electromyographic coherence and phase synchronization analysis between antagonistic elbow muscles. Exp. Brain Res. 233, 971-982 (2014).

28. Danna-Dos Santos, A. et al. Influence of fatigue on hand muscle coordination and EMG-EMG coherence during three-digit grasping. J. Neurophysiol. 104, 3576-3587 (2010).

29. Ushiyama, J. et al. Muscle fatigue-induced enhancement of corticomuscular coherence following sustained submaximal isometric contraction of the tibialis anterior muscle. J. Appl. Physiol. 110, 1233-1240 (2011).

30. McManus, L., Hu, X., Rymer, W. Z., Suresh, N. L. \& Lowery, M. M. Muscle fatigue increases beta-band coherence between the firing times of simultaneously active motor units in the first dorsal interosseous muscle. J. Neurophysiol. 115, 2830-2839 (2016).

31. Boonstra, T. W. Cortical adaptations during muscle fatigue: The role of sensorimotor oscillations. Acta Physiol. 220, 307-309 (2017).

32. Hatton, A. L., Menant, J. C., Lord, S. R., Lo, J. C. M. \& Sturnieks, D. L. The effect of lower limb muscle fatigue on obstacle negotiation during walking in older adults. Gait Posture 37, 506-510 (2013).

33. Helbostad, J. L., Leirfall, S., Moe-Nilssen, R. \& Sletvold, O. Physical fatigue affects gait characteristics in older persons. J. Gerontol. Ser. A Biol. Sci. Med. Sci. 62, 1010-1015 (2007).

34. Barbieri, F. A. et al. Interactions of age and leg muscle fatigue on unobstructed walking and obstacle crossing. Gait Posture 39, 985-990 (2014).

35. Santos, P. C. R. et al. Minimal effects of age and prolonged physical and mental exercise on healthy adults' gait. Gait Posture 74, 205-211 (2019).

36. Morrison, S. et al. Walking-induced fatigue leads to increased falls risk in older adults. J. Am. Med. Dir. Assoc. 17, 402-409 (2016).

37. Santos, P. C. R. et al. Effects of experimentally induced fatigue on healthy older adults' gait: A systematic review. PLoS One 14, e0226939 (2019). 
38. Roldán-Jiménez, C., Bennett, P. \& Cuesta-Vargas, A. I. Muscular activity and fatigue in lower-limb and trunk muscles during different sit-to-stand tests. PLoS One 10, e0141675 (2015).

39 Spedden, M. E., Nielsen, J. B. \& Geertsen, S. S. Oscillatory corticospinal activity during static contraction of ankle muscles is reduced in healthy old versus young adults. Neural Plast. 2018, 20 (2018).

40. Yoshida, T., Masani, K., Zabjek, K., Chen, R. \& Popovic, M. R. Dynamic cortical participation during bilateral, cyclical ankle movements: Effects of aging. Sci. Rep. 7, 1-11 (2017).

41. Nielsen, J. B. et al. Corticospinal transmission to leg motoneurones in human subjects with deficient glycinergic inhibition. J. Physiol. 544, 631-640 (2002).

42. Jensen, P. et al. Using corticomuscular and intermuscular coherence to assess cortical contribution to ankle plantar flexor activity during gait. J. Mot. Behav. 51, 668-680 (2019).

43. Bayram, M. B., Siemionow, V. \& Yue, G. H. Weakening of corticomuscular signal coupling during voluntary motor action in aging. J. Gerontol. A. Biol. Sci. Med. Sci. 70, 1037-1043 (2015).

44. Laine, C. M., Martinez-Valdes, E., Falla, D., Mayer, F. \& Farina, D. Motor neuron pools of synergistic thigh muscles share most of their synaptic input. J. Neurosci. 35, 12207-12216 (2015).

45. Ivanenko, Y. P., Poppele, R. E. \& Lacquaniti, F. Five basic muscle activation patterns account for muscle activity during human locomotion. J. Physiol. 556, 267-282 (2004).

46. Roeder, L., Boonstra, T. W., Smith, S. S. \& Kerr, G. K. Dynamics of corticospinal motor control during overground and treadmill walking in humans. J. Neurophysiol. 120, 1017-1031 (2018).

47. Halliday, D. M. et al. Functional coupling of motor units is modulated during walking in human subjects. J. Neurophysiol. 89, 960-968 (2003).

48. The Editorial Board of The Journal of Neuroscience. Consideration of sample size in neuroscience studies. J. Neurosci. 40, 40764077 (2020).

49. Ward, N. J., Farmer, S. F., Berthouze, L. \& Halliday, D. M. Rectification of EMG in low force contractions improves detection of motor unit coherence in the beta-frequency band. J. Neurophysiol. 110, 1744-1750 (2013).

50. Halliday, D. M. \& Farmer, S. F. On the need for rectification of surface EMG. J. Neurophysiol. 103, 3547-3547 (2010).

51. Boonstra, T. W. \& Breakspear, M. Neural mechanisms of intermuscular coherence: Implications for the rectification of surface electromyography. J. Neurophysiol. 107, 796-807 (2012).

52. Jensen, P. et al. Increased central common drive to ankle plantar flexor and dorsiflexor muscles during visually guided gait. Physiol. Rep. 6, el3598 (2018).

53. Farina, D., Negro, F. \& Jiang, N. Identification of common synaptic inputs to motor neurons from the rectified electromyogram. J. Physiol. 591, 2403-2418 (2013).

54. Hollman, J. H. et al. A comparison of variability in spatiotemporal gait parameters between treadmill and overground walking conditions. Gait Posture 43, 204-209 (2016).

55. Dingwell, J. B., Cusumano, J. P., Cavanagh, P. R. \& Sternad, D. Local dynamic stability versus kinematic variability of continuous overground and treadmill walking. J. Biomech. Eng. 123, 27-32 (2001).

56 Lee, S. J. \& Hidler, J. Biomechanics of overground vs treadmill walking in healthy individuals. J. Appl. Physiol. 104, 747-755 (2008).

57. World Medical Association Declaration of Helsinki. JAMA 310, 2191 (2013).

58. Tombaugh, T. N. \& McIntyre, N. J. The mini-mental state examination: A comprehensive review. J. Am. Geriatr. Soc. 40, 922-935 (1992).

59. Guralnik, J. M., Ferrucci, L., Simonsick, E. M., Salive, M. E. \& Wallace, R. B. Lower-extremity function in persons over the age of 70 years as a predictor of subsequent disability. N. Engl. J. Med. 332, 556-562 (1995).

60. Smets, E. M., Garssen, B., Bonke, B. \& De Haes, J. C. The Multidimensional Fatigue Inventory (MFI) psychometric qualities of an instrument to assess fatigue. J. Psychosom. Res. 39, 315-325 (1995).

61. Goldberg, E. J., Kautz, S. A. \& Neptune, R. R. Can treadmill walking be used to assess propulsion generation?. J. Biomech. 41, $1805-1808$ (2008).

62. Krasovsky, T., Lamontagne, A., Feldman, A. G. \& Levin, M. F. Effects of walking speed on gait stability and interlimb coordination in younger and older adults. Gait Posture 39, 378-385 (2014).

63. Kang, H. G. \& Dingwell, J. B. Effects of walking speed, strength and range of motion on gait stability in healthy older adults. J. Biomech. 41, 2899-2905 (2008).

64. Hermens, H. J., Freriks, B., Disselhorst-Klug, C. \& Rau, G. Development of recommendations for SEMG sensors and sensor placement procedures. J. Electromyogr. Kinesiol. 10, 361-374 (2000).

65. Borg, D. N., Costello, J. T., Bach, A. J. \& Stewart, I. B. Perceived exertion is as effective as the perceptual strain index in predicting physiological strain when wearing personal protective clothing. Physiol. Behav. 169, 216-223 (2017).

66 Buurke, T. J. W., Lamoth, C. J. C., Vervoort, D., van der Woude, L. H. V. \& den Otter, R. Adaptive control of dynamic balance in human gait on a split-belt treadmill. J. Exp. Biol. 221, jeb.74896 (2018).

67. Nandi, T., Hortobágyi, T., van Keeken, H. G., Salem, G. J. \& Lamoth, C. J. C. Standing task difficulty related increase in agonistagonist and agonist-antagonist common inputs are driven by corticospinal and subcortical inputs respectively. Sci. Rep. 9, 1-12 (2019).

68. Amjad, A. M., Halliday, D. M., Rosenberg, J. R. \& Conway, B. A. An extended difference of coherence test for comparing and combining several independent coherence estimates: Theory and application to the study of motor units and physiological tremor. J. Neurosci. Methods 73, 69-79 (1997).

69. Halliday, D. M. \& Rosenberg, J. R. On the application, estimation and interpretation of coherence and pooled coherence. J. Neurosci. Methods 100, 173-174 (2000).

70. Cohen, J. Statistical Power Analysis for the Behavioral Sciences (Lawrence Erlbaum Associates, Hillsdale, 1988).

71. Faul, F., Erdfelder, E., Lang, A.-G. \& Buchner, A. G*Power 3: A flexible statistical power analysis program for the social, behavioral, and biomedical sciences. Behav. Res. Methods 39, 175-191 (2007)

\section{Acknowledgements}

We thank Nick Fennema and Inge Kenter for recruiting the participants and helping with testing. We are grateful for the expert technical assistance provided by Wim Kaan, Anniek Heerschop, Dirk van der Meer, and Emyl Smid. This work was partially supported by Coordenação de Aperfeiçoamento de Pessoal de Nível Superior-Brasil (CAPES) [PCRS, LTBG and FAB, Finance Code 001], the Conselho Nacional de Desenvolvimento Científico e Tecnológico (CNPq) [LTBG-142057/2017-7; 309045/2017-7], and by Graduate School of Medical Science [PCRS]. Funding agencies did not influence the content of the manuscript.

\section{Author contributions}

P.C.R.S., C.J.C.L., F.A.B., I.Z., L.T.B.G. and T.H. conceptualized and designed the study. P.C.R.S. was responsible for the acquisition and analysis of the data. P.C.R.S., C.J.C.L. and T.H. interpreted the results. P.C.R.S. drafted 
the first version of the manuscript. C.J.C.L., F.A.B., I.Z. and T.H. revised the article critically for important intellectual content. P.C.R.S., C.J.C.L., F.A.B., I.Z., L.T.B.G., and T.H. read and approved the final version and agreed to be accountable for all aspects related to the accuracy or integrity of the work.

\section{Competing interests}

The authors declare no competing interests.

\section{Additional information}

Supplementary information is available for this paper at https://doi.org/10.1038/s41598-020-72839-1.

Correspondence and requests for materials should be addressed to P.C.R.S.

Reprints and permissions information is available at www.nature.com/reprints.

Publisher's note Springer Nature remains neutral with regard to jurisdictional claims in published maps and institutional affiliations.

(c) (i) Open Access This article is licensed under a Creative Commons Attribution 4.0 International License, which permits use, sharing, adaptation, distribution and reproduction in any medium or format, as long as you give appropriate credit to the original author(s) and the source, provide a link to the Creative Commons licence, and indicate if changes were made. The images or other third party material in this article are included in the article's Creative Commons licence, unless indicated otherwise in a credit line to the material. If material is not included in the article's Creative Commons licence and your intended use is not permitted by statutory regulation or exceeds the permitted use, you will need to obtain permission directly from the copyright holder. To view a copy of this licence, visit http://creativecommons.org/licenses/by/4.0/.

(c) The Author(s) 2020 